Disclosure(s) Nothing to disclose

\section{P86 TREATMENT MODALITIES IN PERIPHERAL FACIAL NERVE PALSY IN CHILDREN AND ADOLESCENTS}

1,2J Samardzic*, ${ }^{3} \mathrm{~B}$ Kukolj, ${ }^{3} \mathrm{M}$ Jovanovic, ${ }^{4} \mathrm{O}$ Djuric, ${ }^{5} \mathrm{~J}$ Jancic. ${ }^{1}$ Institute of Pharmacology, Clinical Pharmacology and Toxicology, Medical Faculty, University of Belgrade, Belgrade, Serbia; ${ }^{2}$ Division of Paediatric Pharmacology and Pharmacometrics, University of Basel Children's Hospital, Basel, Switzerland; ${ }^{3}$ Medical Faculty, University of Belgrade; ${ }^{4}$ Institute of Epidemiology, Medical Faculty, University of Belgrade; ${ }^{5}$ Clinic of Neurology and Psychiatry for Children and Youth, Medical Faculty, University of Belgrade, Belgrade, Serbia

\subsection{6/archdischild-2019-esdppp.124}

Background Bell's palsy is the most common type of peripheral facial palsy in pediatrics. Recent studies strongly support the combined therapy with corticosteroids (CS), antiviral drugs and vitamins B. Our study aims to assess the effectiveness of proposed therapeutic modalities, including the relation between the patients' recovery and their age, etiological factors and applied treatment.

Methods The retrospective analysis involved 88 patients (52 females/36 males), between 18 months and 18 years old; the average age was 11.7 years. Data was obtained from the documentation of patients hospitalized at the Clinic of Neurology and Psychiatry for Children and Youth in Belgrade, from 2000 to 2017. House Brackmann's scale was used for the assessment of disease course and outcome. Pearson's $\chi 2$ test, Friedman's test and general linear model were applied for statistical data processing.

Results The majority of patients were treated with combined CS/vitamins (42.0\%) and CS/antiviral/vitamins (17.0\%), whereas CS only received $22.7 \%$ of patients, all with an appropriate physical treatment. The group of idiopathic paralysis makes $62.5 \%$, while the incidence of symptomatic paralysis is $37.5 \%$; however, the recovery rate between these groups has not been shown $(\mathrm{p}=0.309)$. Patients received CS therapy were divided into 4 groups: CS only, CS+antiviral, CS+vitaamins $\mathrm{B}$, and $\mathrm{CS}+$ antiviral+vitamins $\mathrm{B}$. The statistically significant recovery was registered in each group $(\mathrm{p}<0.001)$; however, no difference was found between the groups in term of recovery rate $(p=865)$. For the assessment of recovery period in relation to the age, the obtained $\mathrm{p}$ value was 0.054 , a borderline level, suggesting a faster recovery of children at younger age.

Conclusion The acute one-sided mimic musculature weakness is mostly idiopathic. The effectiveness of the CS therapy was strongly supported, suggesting CS as a core treatment for the Bell's palsy. It has been shown the faster recovery of children at younger age.

Disclosure(s) Nothing to disclose

\section{P87 THE SWISS ASSOCIATION OF PERINATAL PHARMACOLOGY, SAPP: GOALS}

L Schenkel* ${ }^{*}$ U von Mandach, The Editorial Board of the SAPP. Obstetrics, University Hospital of Zurich, Zürich, Switzerland

\subsection{6/archdischild-2019-esdppp.125}

Background The medicine of pregnant women practices to a targeted personalized approach, tailored to the specific characteristics and needs the implications of interdisciplinary work between healthcare stakeholders. Perinatal pharmacology comprises the impact of substances (drugs, medications and others) in pregnant women, nursing mothers, the unborn child, the premature, the newborn baby and the breastfed baby. Most of the drugs are off-label used. In this field grand challenge for Frontiers in Medicine emphasizes the importance of translational medicine.

Aim The primary goal of SAPP has always been and remains the same: it is the link between medicine and pharmacy, between practice, clinic, research and health authorities, in order to increase the safety of medicines in the population of pregnant and breastfeeding women and their newborns. ${ }^{1}$

Methods On December 6, 2007, an interdisciplinary team of 8 physicians and pharmacists founded the Swiss Association of Perinatal Pharmacology, SAPP. It collects and promotes new findings in the field of perinatal pharmacology.

Results Specialists from all areas of perinatal pharmacology work together in a scientific committee to develop and update evidence-based principles for work in everyday clinical practice (hospitals, doctor's surgeries, pharmacies). Today, around 200 members benefit from this, who can orient themselves in regular further training courses and basic documents (monographs of active substances, therapy recommendations based on original literature). The SAPP thus closes the gap resulting from the predominant off-label use and the resulting lack of information on drugs in this population.

Conclusion The primary objective of SAPP has been achieved - it provides guidance for the practioners in the broad field of perinatal pharmacology and bridges the gap caused by the lack of drug approvals in this population. Long-term survival will be ensured by measures anchored in law.

\section{REFERENCE}

1. www.sappinfo.ch

Disclosure(s) Nothing to disclose

\section{P88 THE POTENTIAL IMPACT OF HEMATOCRIT CORRECTION ON EVALUATION OF TACROLIMUS TARGET EXPOSURE IN PEDIATRIC KIDNEY TRANSPLANT PATIENTS}

\begin{abstract}
${ }^{1} \mathrm{~A}$ Schijvens ${ }^{*},{ }^{1} \mathrm{~F}$ van Hesteren, ${ }^{1} \mathrm{M}$ Cornelissen, ${ }^{1} \mathrm{C}$ Bootsma-Robroeks, ${ }^{2} \mathrm{R}$ Brüggemann, ${ }^{2} \mathrm{D}$ Burger, ${ }^{3,4} \mathrm{~S}$ de Wildt, ${ }^{1} \mathrm{M}$ Schreuder, ${ }^{2} \mathrm{R}$ ter Heine. ${ }^{1}$ Pediatric Nephrology, Radboud University Medical Center, Radboud Institute for Molecular Life Sciences, Amalia Children's Hospital; ${ }^{2}$ Pharmacy; ${ }^{3}$ Department of Pharmacology and Toxicology, Radboud University Medical Center, Radboud Institute for Health Sciences, Nijmegen; ${ }^{4}$ Intensive Care and Department of Pediatric Surgery, Erasmus MC Sophia Children's Hospital, Rotterdam, The Netherlands
\end{abstract}

\subsection{6/archdischild-2019-esdppp.126}

Background Tacrolimus is an important immunosuppressive agent with high intra- and interindividual pharmacokinetic variability and a narrow therapeutic index. As tacrolimus extensively accumulates in erythrocytes, hematocrit is a key factor in the interpretation of tacrolimus whole blood concentrations. However, as hematocrit values in pediatric kidney transplant patients are highly variable after kidney transplantation, translating whole blood concentration targets without taking hematocrit into consideration, is theoretically incorrect. The aim of this study is to evaluate the potential impact of hematocrit correction on tacrolimus target exposure in pediatric kidney transplant patients.

Methods Data were obtained from 36 pediatric kidney transplant patients. 255 tacrolimus whole blood samples were 